\title{
SINGLE-SERVER QUEUEING SYSTEMS WITH UNIFORMLY LIMITED QUEUEING TIME
}

\author{
D. J. DALEY \\ (received 8 January 1964)
}

\begin{abstract}
Summary
The paper considers the queueing system $G I / G / 1$ with a type of customer impatience, namely, that the total queueing-time is uniformly limited. Using Lindley's approach [10], an integral equation for the limiting waitingtime distribution is derived, and this is solved explicitly for $M / G / 1$ using an expansion of the Pollaczek-Khintchine formula. It is also solved, in principle for $E_{j} / G / 1$, and explicitly for $E_{j} / E_{k} / 1$. A duality noted between $G I_{A(x)} / G_{B(x)} / 1$ and $G I_{B(x)} / G_{A(x)} / 1$ relates solutions for $G I / E_{k} / 1$ to $E_{k} / G / 1$. Finally the equation for the busy period in $G I / G / 1$ is derived and related to the no-customer-loss distribution and dual distributions.
\end{abstract}

\section{Introduction}

This paper discusses the following single-server queueing system with a certain type of customer impatience. The customers $C_{0}, C_{1}, \cdots, C_{n}, \cdots$ arrive at instants $T_{0}, T_{1}, \cdots, T_{n}, \cdots$, and for complete service require attention for times $s_{0}, s_{1}, \cdots, s_{n}, \cdots$, the server attending them in order of arrival. The sequences $\left\{t_{n}\right\}$ of inter-arrival times $t_{n}=T_{n+1}-T_{n}$, $n=0,1,2, \cdots$, and $\left\{s_{n}\right\}$ are assumed to be independent sequences of independent non-negative random variables with distribution functions $A(x)$ and $B(x)$ respectively, $A(0)=0$. The waiting-time $w_{n}$ of customer $C_{n}$ is the time between his arrival at $T_{n}$ and the instant where he starts to receive service; if the server is free at $T_{n}$, the service of $C_{n}$ begins immediately.

The customer impatience is such that the total time spent in the system by each customer is uniformly limited by a constant $K$. Because $\operatorname{Pr}\left(t_{n}>0\right)=1-A(0)=1$ and the customers are served in order of arrival, every waiting-time $w_{n}$ is strictly less than $K$ (with probability one), and customers leaving the system impatiently do so by cutting short the time spent with the server. Thus, $C_{n}$ receives service for a time $\min \left(s_{n}, K-w_{n}\right)$. 
Using the technique first developed by Lindley [10], it follows that the waiting-time sequence $\left\{w_{n}\right\}$ satisfies

$$
\begin{aligned}
w_{n+1} & =\left[w_{n}+\min \left(s_{n}, K-w_{n}\right)-t_{n}\right]^{+} \\
& =\left[\min \left(w_{n}+s_{n}, K\right)-t_{n}\right]^{+}
\end{aligned}
$$

where $[x]^{+}=\max (0, x) .\left\{w_{n}\right\}$ is thus a Markov chain with state space $\Omega=[0, K]$.

Define $W_{n}(x)=\operatorname{Pr}\left(w_{n} \leqq x\right)$ as the distribution function of $w_{n}$, $P,(t)$ as the probability that at time $t$ there are $j$ customers in the system, and write $W(x)=\lim _{n \rightarrow \infty} W_{n}(x)$ and $P_{j}=\lim _{t \rightarrow \infty} P_{j}(t)$ when the limits exist.

Barrer [2] and Kovalenko [8] found both the distribution $\left\{P_{j}\right\}$ and $W(x)$ for the models $M / M / 1$ and $M / M / s$ respectively by means of birthand-death equations (Barrer) and a multi-dimensional Markov process (Kovalenko). Kovalenko claimed that his results disagreed with Barrer's in the single-server case $(s=1)$, but it appears to be a misunderstanding of notation; our results below concerning $W(x)$ agree with both. Barrer [1] also discussed $M / M / 1$ with random order of service. The model has a finite dam analogue (see $\S 2$ below) and in this context Prabhu [14] and Ghosal [5] have solved the system $E_{j} / D / 1$ explicitly. Kovalenko [9] has used 'Takacs' [17] approach through the virtual waiting-time to study the queue $M / G / 1$ with a general scheme of customer impatience. Our model is a special case of this general scheme, and equation (9) below for $M / G / 1$ may be deduced from Kovalenko's general equation.

\section{Analogous models}

A finite dam model due to Moran [13] and a two-barrier model for the moving server problem also lead to equation (1).

The storage $Z_{t}$ of a dam of finite capacity $K$ is defined for discrete time $t=0,1,2, \cdots$. In the interval $(t, t+1)$ suppose an amount $X_{t}$ flows into the dam, filling it to the level $\min \left(Z_{t}+X_{t}, K\right)$, any water in excess of the dam's capacity $K$ being lost over the spillway. Just before the instant $t+1$ a demand for an amount $Y_{t}$ occurs, and this is met as fully as possible by the release of the quantity $\min \left[Y_{t}, \min \left(Z_{t}+X_{t}, K\right)\right]=$ $\min \left(Y_{t}, Z_{t}+X_{t}, K\right)$. This leaves an amount $Z_{t+1}$ in the dam, where

$$
\begin{aligned}
Z_{t+1} & =\min \left(Z_{t}+X_{t}, K\right)-\min \left(Y_{t}, Z_{t}+X_{t}, K\right) \\
& =\left[\min \left(Z_{t}+X_{t}, K\right)-Y_{t}\right]^{+} .
\end{aligned}
$$

This equation is of the same form as $(1)$ if $\left\{X_{t}\right\}$ and $\left\{Y_{t}\right\}$ are assumed to be independent sequences of independent non-negative random variables with distribution functions $B(x)$ and $A(x)$ respectively. 
The model is most easily visualized by supposing the dam to be located in a region with distinct wet and dry seasons; then $Z_{t}$ is the lowest level of the dam, and occurs at the end of the dry season. $\left\{Z_{t}\right\}$ remains a Markov chain if we allow the demand $Y_{t}$ to depend on the input $X_{t}$ immediately preceding it.

In the moving server model we propose there is an assembly line moving at uniform unit speed with items requiring service spaced along it at intervals $s_{n}$. The single server attends the items for times $t_{n}$, where $\left\{s_{n}\right\}$ and $\left\{t_{n}\right\}$ are independent sequences of independent non-negative random variables with distribution functions $B(x)$ and $A(x)$ respectively. The server may start on a given item only when it has passed a certain point called the entrance gate, and if he has not finished serving an item by the time it reaches a point $K$ further along the line called the exit gate, the whole line is stopped and a disruption occurs. As soon as he finishes each item, the server instantaneously moves back along the line to the next item or to the entrance gate, whichever the nearer.

Define $u_{n}$ as the distance from the exit gate at which service of the $n$th item finishes. Then

$$
u_{n+1}=\left[\min \left(u_{n}+s_{n}, K\right)-t_{n}\right]^{+}
$$

which is of the same form as (1).

The function investigated in the literature [6] and [12] is $\left\{p_{n}(x)\right\}$, the probability distribution of serving exactly $n$ items before disruption, given that service on the first item begins when it is a distance $x$ away from the exit gate (i.e., the absorbing barrier). The entrance gate we have introduced above is not included in the model used in [6] and [12], and the one-barrier model is therefore analogous to an infinite dam or the standard queueing system $(K=\infty)$. Since $A(x)$ was assumed to be negative exponential, these earlier workers could exploit the analogy with Takacs' work [17] on the busy period in $M / G / 1$.

\section{Dual of the waiting-time}

In the model under consideration there exists a sequence $\left\{v_{n}\right\}$ of random variables which is the dual of $\left\{w_{n}\right\}$ in the sense that the functional dependence of $\left\{w_{n}\right\}$ on $\left\{s_{n}\right\}$ and $\left\{t_{n}\right\}$ is the same as that of $\left\{v_{n}\right\}$ on $\left\{t_{n}\right\}$ and $\left\{s_{n+1}\right\}$.

Put $v_{n}$ equal to the difference between $K$, the maximum total queueingtime for any customer $C_{n}$, and his actual queueing-time $\min \left(w_{n}+s_{n}, K\right)$, i.e.,

$$
\begin{aligned}
v_{n} & =K-\min \left(w_{n}+s_{n}, K\right) \\
& =\left[K-w_{n}-s_{n}\right]^{+} .
\end{aligned}
$$

Recall (1): 


$$
\begin{aligned}
w_{n+1} & =\left[\min \left(w_{n}+s_{n}, K\right)-t_{n}\right]^{+} \\
& =\left[K-v_{n}-t_{n}\right]^{+}
\end{aligned}
$$

so

$$
\begin{aligned}
v_{n+1} & =\left[K+\min \left(v_{n}+t_{n}-K, 0\right)-s_{n+1}\right]^{+} \\
& =\left[\min \left(v_{n}+t_{n}, K\right)-s_{n+1}\right]^{+}
\end{aligned}
$$

which is the dual of (1) and proves our assertion.

Define the distribution function of $v_{n}$ by $V_{n}(x)=\operatorname{Pr}\left(v_{n} \leqq x\right)$, noting that $V_{n}(K)=1$ for all $n=1,2, \cdots$ (and indeed, $W_{n}(K)=1$ also). Using $(2), V_{n}(x)$ and $W_{n}(x)$ are related by

$$
V_{n}(x)=1-\int_{0}^{K-x} W_{n}(K-x-y-0) d B(y) .
$$

Similarly,

$$
W_{n+1}(x)=i-\int_{0}^{K-x} V_{n}(K-x-y-0) d A(y)
$$

whence the following result is evident:

LEMMA 1. $\operatorname{Lim}_{n \rightarrow \infty} W_{n}(x)=W(x)$ exists if and only if $\lim _{n \rightarrow \infty} V_{n}(x)=V(x)$ exists, in which case they are related by

$$
V(x)=1-\int_{0}^{K-x} W(K-x-y-0) d B(y) .
$$

and its dual

$$
W(x)=1-\int_{0}^{K-x} V(K-x-y-0) d A(y) .
$$

The importance of (3) and (4) is that in any given algebraic situation, $W(x)$ (for example) may be more simply found by the techniques outlined below whereas $V(x)$ may be the function of greater interest.

In terms of the finite dam model of $\S 2$, the interpretation of the dual $\left\{V_{t}\right\}$ of $\left\{Z_{t}\right\}$ is as the volume of the dam remaining to be filled at the end of the wet season.

\section{Integral equation for the stationary waiting-time distribution}

THEOREM 1. Unless $s_{n}=t_{n}=$ constant $<K$, a unique stationary waitingtime distribution function $W(x)=\lim _{n \rightarrow \infty} W_{n}(x)$ exists, is independent of the initial state of the system, and satisfies the equation for $0 \leqq x \leqq K$,

$$
W(x)=1-A(K-x-0)+\int_{u=x}^{K-0} d A(u-x) \int_{0}^{u} W(u-y) d B(y) .
$$

Proof. From (1), for $x \geqq 0$, 


$$
\begin{aligned}
W_{n+1}(x) & =\operatorname{Pr}\left[\min \left(w_{n}+s_{n}, K\right)-t_{n} \leqq x\right] \\
& =\operatorname{Pr}\left[t_{n} \geqq K-x\right]+\operatorname{Pr}\left[w_{n}+s_{n} \leqq x+t_{n}<K\right] \\
& =1-A(K-x-0)+\int_{u=x}^{K-0} d A(u-x) \int_{0}^{u} W_{n}(u-y) d B(y)
\end{aligned}
$$

which determines the entire sequence $\left\{W_{n}(x)\right\}$ given the initial $w_{0}$ (or, $\left.W_{0}(x)\right)$. (5) is the obvious formal limit of $(6)$; we now prove the existence of $W(x)$ with the properties asserted.

If $s_{n}=t_{n}=$ constant $=M$ say, then if $M<K, w_{n}=w_{0}$ if $0 \leqq w_{0}<K-M$, while for other $w_{0}, w_{n}=K-M$; otherwise $(M \geqq K)$, $w_{n}=0$ for all $n=1,2, \cdots$ irrespective of $w_{0}$. These assertions follow directly from (1).

Define

$$
\begin{aligned}
& x_{a}=\min (K, \text { g.l.b. }\{x: A(x)>0\}), \\
& x_{A}=\text { l.u.b. }\{x: A(x)<1, x \leqq K\}
\end{aligned}
$$

and, similarly, $x_{b}, x_{B}$ in terms of $B(x)$. Excluding the case $x_{a}=x_{A}=x_{n}=x_{B}$ which means either $s_{n}=t_{n}=$ constant $<K$ which has been discussed already, or $x_{a}=\cdots=x_{B}=K$ in which case the theorem is trivial, there are two cases: (i) $x_{A}>x_{b}$; (ii) $x_{A} \leqq x_{b}$ and $x_{a} \neq x_{B}$, implying $x_{B}>x_{a}$. (ii) is thus the dual of (i), so by Lemma 1 it suffices to prove the theorem in case (i).

Choose positive $\alpha, \beta, \gamma$ such that $\alpha+\beta+\gamma=1$, and with $\Delta=x_{A}-x_{b}$, set $a=A\left(x_{A}-\alpha \Delta\right), b=B\left(x_{b}+\beta \Delta\right)$. Then by definition of $x_{A}$ and $x_{b}$, $a<1$ and $b>0$, so $c=(1-a) b>0$. For any positive integer $n$ and any $\xi \in \Omega=[0, K]$,

$$
\begin{aligned}
\operatorname{Pr}\left(w_{n+1} \leqq[\xi-\gamma \Delta]^{+} \mid w_{n}\right. & =\xi)=\operatorname{Pr}\left(\min \left(\xi+s_{n}, K\right)-t_{n} \leqq[\xi-\gamma \Delta]^{+}\right) \\
& \geqq \operatorname{Pr}\left(t_{n}>x_{A}-\alpha \Delta, s_{n} \leqq x_{b}+\beta \Delta\right) \\
& =(1-a) b=c .
\end{aligned}
$$

Similarly, $\operatorname{Pr}\left(w_{n+2} \leqq[\xi-2 \gamma \Delta]+\mid w_{n}=\xi\right) \geqq c^{2}$, and so on. Let $N$ be the least integer greater than $K / \gamma \Delta$, so that $N$ is finite. Then $[\xi-N \gamma \Delta]^{+}=0$ for $\xi \in \Omega$, and hence, for arbitrary positive $n$ and arbitrary $\xi \in \Omega$,

$$
\operatorname{Pr}\left(w_{n+N}=0 \mid w_{n}=\xi\right) \geqq c^{N}>0 .
$$

Define a measure $\boldsymbol{v}$ on the Borel field $\mathscr{B}$ over $\Omega$ by $\boldsymbol{v}(A)=0$ if $0 \notin A$, $\nu(A)=1$ if $0 \in A$, where $A \in \mathscr{B}$. Then $v(\Omega)=1<\infty$, and for $0<\varepsilon<c^{N}$, if $\gamma(A) \leqq \varepsilon$ (so $0 \notin A$ ), the $N$-step transition probability

$$
p^{N}(\xi, A) \leqq p^{N}(\xi, \Omega-0) \leqq 1-c^{N}<1-\varepsilon,
$$

so Doeblin's condition (Doob [3], pp. $192 \mathrm{ff}$, and pp. 197-8 in particular) is satisfied. Therefore a unique stationary probability measure on $\mathscr{B}$ exists, 
it is independent of the initial state of the system, and from (6), it satisfies (5). This proves the theorem.

Remarks. 1. Doeblin's condition leads in fact to exponentially fast convergence. We could alternatively have used a recent result of Finch [4] to prove the theorem, and, indeed, a special case of our result is one of his examples for which he has calculated the exponential bounds.

2. For $w_{0}=0$ it is easy to show from (6) by forming an integral equation in $\left[W_{n+1}(x)-W_{n}(x)\right]$ that $W_{n}(x)$ converges to $W(x)$ monotonically from above. This monotone convergence starting from $w_{0}=0$ also follows from recent work of Loynes [11] from whose Lemma 1 there may be proved the stronger result that monotone convergence exists when $\left\{U_{n}\right\}=\left\{\left(s_{n}, t_{n}\right)\right\}$ is a stationary sequence of random variables. That is, the assumptions concerning $\left\{s_{n}\right\}$ and $\left\{t_{n}\right\}$ are weakened, and stationarity of $\left\{U_{n}\right\}$ is sufficient.

\section{Solution for the system $M / G / 1$}

In a system in which the inter-arrival times have the negative exponential distribution

we obtain

$$
A(x)=1-e^{-\lambda x}, \quad x \geqq 0, \lambda>0
$$

THEOREM 2. The stationary waiting-time distribution function $W(x)$ in the queueing system $M / G / 1$ with uniformly limited total queueing-time is given by

$$
W(x)=\frac{\sum_{n=0}^{\infty} \int_{0-}^{x} \frac{[-\lambda(x-u)]^{n}}{n !} e^{\lambda(x-u)} d B^{n *}(u)}{\sum_{n=0}^{\infty} \int_{0-}^{K} \frac{[-\lambda(K-u)]^{n}}{n !} e^{\lambda(K-u)} d B^{n *}(u)}, \quad 0 \leqq x \leqq K .
$$

Proof. Substituting for $A(x)$ in (5) yields that for $0 \leqq x \leqq K$,

$$
W(x)=e^{-\lambda(K-u)}+\int_{x}^{K} \lambda e^{-\lambda(u-x)} d u \int_{0}^{u} W(u-y) d B(y)
$$

$W(x)$ is therefore differentiable on $[0, K]$ with

$$
W^{\prime}(x)=\lambda W(x)-\lambda \int_{0}^{x} W(x-y) d B(y) .
$$

Consider now the following equation for a function $F(x), x \geqq 0$ :

$$
F^{\prime}(x)=\lambda F(x)-\lambda \int_{0}^{x} F(x-y) d B(y)
$$

(the existence of such a function is proved below). $F(x) \equiv 0$ is a trivial solution of (9). If any non-trivial solution exists, then its Laplace-Stieltjes transform, 


$$
\phi(\theta)=\int_{0-}^{\infty} e^{-\theta x} d F(x)
$$

if it exists, satisfies

$$
\phi(\theta)-F(0)=(\lambda / \theta)[\phi(\theta)-\beta(\theta) \phi(\theta)]
$$

where

$$
\beta(\theta)=\int_{0-}^{\infty} e^{-\theta x} d B(x)
$$

is the Laplace-Stieltjes transform of $B(x)$. Rewriting the equation,

$$
\phi(\theta)=\frac{\theta F(0)}{\theta-\lambda+\lambda \beta(\theta)} .
$$

This formula, in the case where $F(x)$ is a distribution function, is the wellknown Pollaczek-Khintchine formula for the standard $(K=\infty)$ queueing system $M / G / 1$ with $\rho=E s_{n} / E t_{n}<1$, when $F(0)=1-\rho$ (see e.g. Kendall [7]). It is known further that for this case (see e.g. Prabhu [15]) the series expansion below for $\phi(\theta)$ is valid:

$$
\frac{1}{\theta-\lambda+\lambda \beta(\theta)}=\frac{1}{\theta-\lambda} \sum_{n=0}^{\infty}\left(\frac{\lambda}{\lambda-\theta}\right)^{n} \beta^{n *}(\theta)
$$

where $\beta^{n *}(\theta)$ is the transform of the $n$-fold convolution of $B(x)$ with itself, setting $B^{0 *}(x)=0$ or 1 as $x<$ or $\geqq 0$. The expansion is in fact valid for all $\theta$ in the half-plane $\operatorname{Re}(\theta)>2 \lambda$. It may be inverted term by term there to yield

$$
F(x)=F(0) \sum_{n=0}^{\infty} \int_{0-}^{x} \frac{[-\lambda(x-u)]^{n}}{n !} e^{\lambda(x-u)} d B^{n *}(u)
$$

Now for any $F(0)$, (12) satisfies (9). Determine $F(0)$ by $x=K$ in (12) and $F(K)=1$, and set $W(x)=F(x), 0 \leqq x \leqq K$, so that (8) is then satisfied by $W(x)$ as in (7). The theorem is proved.

For the finite dam model analogue, Prabhu [14] solved the systems $M / D / 1$ and $E_{j} / D / 1$ by a method using repeated substitution, and (7) with $B(x)=0$ or 1 as $x<$ or $\geqq M$ agrees with his solution. Such substitution in (7) is the easiest method of solving (8) when $B(x)$ is deterministic, but when $B(x)$ has a rational transform $\beta(\theta)$, direct inversion of $(10)$ is an easier method, as given in an example shortly.

In the example below and again in $\S 6$ the polynomial

$$
P(z)=(1-\gamma z)^{j}(1+z)^{k}-1
$$

occurs, with $z=\theta / \mu$ and $\gamma=\mu / \lambda$. Theorem 3 contains results concerning $P(z)$ which are in no sense new; the use of Rouche's theorem to prove them is well-established (see e.g. Takacs [18]) and the proof is omitted. 
We recall that the queueing system $E_{j} / E_{k} / 1$ with parameters $\lambda, j$ and $\mu$, $k$ has traffic intensity $\rho=\lambda k / \mu j=k / \gamma j$.

THEOREM 3. If $\rho=k / \gamma j=1$, then $P(z)$ defined by (13) has a double. zero at $z=0$ and all its other $j+k-2$ zeros are distinct. If $\rho \neq 1$, all the $j+k$ zeros of $P(z)$ are distinct and are distributed as follows: if $\rho<1, j$ lie within or on the circle $|1-\gamma z|=1$ and the other $k$ lie within the circle $|1+z|=$ $k(1+\gamma) / \gamma(j+k)$; if $\rho>1, j$ lie within the circle $|1-\gamma z|=k(1+\gamma) / \gamma(j+k)$ and the other $k$ lie within or on the circle $|1+z|=1$.

Example. $M / E_{k} / 1$.

For $B(x)=1-e^{-\mu x} \sum_{,=0}^{k-1}(\mu x)^{i} / i !, \quad x \geqq 0, \quad \mu>0, \quad \beta(\theta)=(1+\theta / \mu)^{-k}$. Substituting in (10),

$$
\frac{\phi(\theta)}{\theta F(0)}=\frac{(1+\theta / \mu)^{k}}{\lambda\left[1-(1-\theta / \lambda)(1+\theta / \mu)^{k}\right]}
$$

where the polynomial in the denominator is a particular case of (13). We shall assume $\rho=\lambda k / \mu \neq 1$, so that by Theorem 3, all the $k+1$ zeros, $\beta_{0}=0, \beta_{1}, \cdots, \beta_{k}$, of the denominator are distinct. The partial fraction expansion

$$
\frac{\phi(\theta)}{\theta F(0)}=\sum_{i=0}^{k} \frac{A_{i}}{\theta-\beta_{i}}
$$

where the constants $A_{i}$ are evaluated by l'Hôpital's rule

$$
\begin{aligned}
A_{i} & =\lim _{\theta \rightarrow \beta_{i}} \frac{\left(\theta-\beta_{i}\right) \phi(\theta)}{\theta F(0)} \\
& =\frac{\beta_{i}+\mu}{(k+1) \beta_{i}+\mu-k \lambda}
\end{aligned}
$$

is therefore valid. We note that using the zeros $\alpha_{0}=0, \alpha_{1}, \cdots, \alpha_{k}$ of $P(z)$ gives the alternative expression for $A_{i}$,

$$
A_{i}=\frac{\alpha_{i}+1}{(k+1) \alpha_{i}+1-\rho} .
$$

Inverting the transforms in (14),

$$
F(x)=F(0) \sum_{i=0}^{k} A_{i} e^{\beta_{i} x}
$$

and on using the boundary condition $F(K)=1$ and identifying $W(x)$ with $F(x)$ on $0 \leqq x \leqq K$,

$$
W(x)=\sum_{i=0}^{k} A_{i} e^{\beta_{i} x} / \sum_{i=0}^{k} A_{i} e^{\beta_{i} K}, \quad 0 \leqq x \leqq K .
$$


With $\rho<1$, the zeros $\beta_{1}, \cdots, \beta_{k}$ have negative real part, so by letting $K \rightarrow \infty$ in (16), the model becomes the standard queueing system $M / G / 1$ with no impatience, $A_{0}=1 /(1-\rho)$ by (15), and hence

$$
W(x)=1+(1-\rho) \sum_{i=1}^{k} A_{\imath} e^{\beta_{i} x}, \quad x \geqq 0,
$$

which is as quoted by Saaty [16], p. 212.

We mention finally that by using transforms of $W(x)($ not $F(x))$, the following moments of the distribution function $W(x)$ may be calculated:

$$
\begin{aligned}
E(w)= & K\left[1-A_{0} W(0)\right]+W(0) \sum_{i=1}^{k}\left(1-e^{\beta_{i} K}\right) A_{i} / \beta_{i} \\
\operatorname{Var}(w)= & W(0) \sum_{i=1}^{k}\left(e^{\beta_{1} K}-1\right) A_{i} / \beta_{i}^{2}-[E(w)]^{2} \\
& -2 K\left[W(0) \sum_{i=1}^{k} A_{i} / \beta_{i}-E(w)\right]-K^{2}\left[1-A_{0} W(0)\right]
\end{aligned}
$$

which for $K \rightarrow \infty$ give

and

$$
E(w)=W(0) \sum_{i=1}^{k} A_{i} / \beta_{i}
$$

$$
\operatorname{Var}(w)=-W(0) \sum_{i=1}^{k} A_{i} / \beta_{i}^{2}-[E(w)]^{2}
$$

where $W(0)=1-\rho$ and the $A_{i}$ 's are given by (15).

\section{Solution for the system $E_{J} / G / 1$}

When the inter-arrival time distribution function is Erlang instead of negative exponential, the same formal processes as used for $M / G / 1$ lead to a solution of the integral equation (5). Some of the steps below need further justification; we shall indicate where this is required.

We write

where

$$
\begin{aligned}
A(x) & =1-e^{-\lambda x} \sum_{i=0}^{j-1}(\lambda x)^{i} / i !, \quad x \geqq 0, \quad \lambda>0 \\
& =\int_{0}^{x} a,(u) d u
\end{aligned}
$$

$$
a_{j}(u)=\lambda e^{-\lambda u}(\lambda u)^{2-1} /(j-1) !, \quad j=1,2,3, \cdots, u \geqq 0 .
$$

It is easy to verify that $a_{j}(u)$ and its derivatives $a_{j}^{(n)}(u)$ satisfy for $n=0,1, \cdots, j-1$,

$$
a_{j}^{(n)}(u)=\lambda^{n} \sum_{\imath=0}^{n}(-)^{2}\left(\begin{array}{l}
n \\
i
\end{array}\right) a_{j-n+\imath}(u)
$$

and 


$$
a_{j-n}(u)=\sum_{i=0}^{n}\left(\begin{array}{l}
n \\
i
\end{array}\right) \lambda^{-i} a_{j}^{(i)}(u) .
$$

Substituting for $A(x)$ in (5), $W(x)$ satisfies for $0 \leqq x \leqq K$,

$$
W(x)=\int_{0}^{\infty} a_{j}(K-x+u) d u+\int_{x}^{K} a_{j}(u-x) d u \int_{0}^{u} W(u-y) d B(y)
$$

showing $W(x)$ to be differentiable. In fact, $W(x)$ is $j$ times differentiable, and for $i=0,1, \cdots, j-1$,

$$
(-)^{i} W^{(i)}(x)=\int_{0}^{\infty} a_{j}^{(i)}(K-x+u) d u+\int_{x}^{K} a_{j}^{(i)}(u-x) d u \int_{0}^{u} W(u-y) d B(y)
$$

with

$$
W(K)=1, W^{(i)}(K)=0, \quad i=1,2, \cdots, j-1,
$$

using (17) with (19) to establish the conditions (20) on the derivatives. Multiplying (19) by $\left(\begin{array}{c}i-1 \\ i\end{array}\right) \lambda^{-i}$, summing over $i=0,1, \cdots, j-1$, and using (18)

$$
\sum_{i=0}^{j-1}\left(\begin{array}{c}
j-1 \\
i
\end{array}\right)(-\lambda)^{-i} W^{(i)}(x)=\lambda e^{-\lambda(K-x)}+\lambda e^{\lambda x} \int_{x}^{K} e^{-\lambda u} d u \int_{0}^{u} W(u-y) d B(y)
$$

Differentiate for the $j$ th time. Then on rearrangement,

$$
\sum_{i=1}^{j}\left(\begin{array}{l}
j \\
i
\end{array}\right)(-\lambda)^{-i} W^{(i)}(x)=\int_{0}^{x} W(x-y) d B(y)-W(x)
$$

We now assume that there exists a function $F(x)$ which coincides with $W(x)$ on $0 \leqq x \leqq K$, so that it satisfies (21) and (20), and that it also satisfies (21) for all positive $x$. Assuming furthermore that the LaplaceStieltjes transform $\phi(\theta)$ of $F(x)$ exists for $\theta$ in some half-plane, we have

$$
\int_{0+}^{\infty} e^{-\theta x} F^{(i+1)}(x) d x=\theta^{i} \phi(\theta)-\sum_{r=0}^{i} \theta^{n} F^{(i \rightarrow)}(0)
$$

This identity enables us to take transforms in (21) (with $W(x)$ replaced by $F(x)$ ) which yields on simplification

$$
\phi(\theta)=\frac{\sum_{r=0}^{j-1} \theta^{-r} F^{(r)}(0) \sum_{i=r+1}^{j}\left(\begin{array}{l}
j \\
i
\end{array}\right)(-\theta / \lambda)^{i}}{(1-\theta / \lambda)^{j}-\beta(\theta)} .
$$

In the case $j=1,(22)$ reduces to $(10)$. Were we to be rigorous, we should now try and find an expansion as we did for (10) in order to justify the assumption of the existence of a function $F(x)$ with the required properties. 
Example. $E_{j} / E_{k} / 2$.

We outline the steps in determining $F(x)$, and hence $W(x)$, from (20) and (22) when

$$
B(x)=1-e^{-\mu x} \sum_{i=0}^{k-1}(\mu x)^{i} / i !
$$

so that $\beta(\theta)=(1+\theta / \mu)^{-k}$. We seek therefore to invert

$$
\frac{\phi(\theta)}{\theta}=\frac{(1+\theta / \mu)^{k} \sum_{r=0}^{j-1} \theta^{-r-1} F^{(r)}(0) \sum_{i=r+1}^{j}\left(\begin{array}{l}
j \\
i
\end{array}\right)(-\theta / \lambda)^{2}}{(1-\theta / \lambda)^{j}(1+\theta / \mu)^{k}-1} .
$$

Assuming $\rho=\lambda k / \mu j \neq 1$ and appealing to Theorem 3 , the $j+k$ zeros $\beta_{i}, i=1,2, \cdots, j+k$, of the denominator are distinct, the numerator is a polynomial of degree $j+k-1$ (at most), so the following partial fraction expansion is valid:

$$
\frac{\phi(\theta)}{\theta}=\sum_{i=1}^{j+k} \frac{B_{i}}{\theta-\beta_{i}}
$$

where the constants $B_{i}$ are obtained by l'Hôpital's rule:

$$
\begin{aligned}
B_{i} & =\frac{\left(1+\beta_{i} / \mu\right) \sum_{r=0}^{j-1} \beta_{i}^{-r-1} F^{(r)}(0) \sum_{g=r+1}^{j}\left(\begin{array}{l}
j \\
s
\end{array}\right)\left(-\beta_{i} / \lambda\right)^{s}}{\left(1-\beta_{i} / \lambda\right)^{j-1}\left[\left(1-\beta_{i} / \lambda\right) k / \mu-\left(1+\beta_{i} / \mu\right) j / \lambda\right]} \\
& =\frac{\left(1+\alpha_{i}\right) \sum_{r=0}^{j-1}\left(\mu \alpha_{i}\right)^{-r-1} F^{(r)}(0) \sum_{s=r+1}^{j}\left(\begin{array}{l}
j \\
s
\end{array}\right)\left(-\gamma \alpha_{i}\right)^{s}}{\left(1-\gamma \alpha_{i}\right)^{j-1}\left[(1-\rho) k / \mu-(k+j) \alpha_{i} / \lambda\right]}
\end{aligned}
$$

using the $j+k$ zeros $\alpha_{i}$ of $P(z)$ defined in (13).

Inverting the transform,

$$
F(x)=\sum_{i=1}^{k} B_{i} e^{\beta_{i} x}
$$

and the derivatives for $n=0,1, \cdots, j-1$ are

$$
F^{(n)}(x)=\sum_{i=1}^{j+k} B_{i} \beta_{i}^{n} e^{\beta_{2} x}
$$

Using the condition (20), we obtain

$$
\begin{aligned}
& \sum_{i=1}^{j+k} B_{i} e^{\beta_{i} K}=1, \\
& \sum_{i=1}^{j+k} B_{i} \beta_{i}^{n} e^{\beta_{i} K}=0, \quad n=1,2, \cdots, j-1 .
\end{aligned}
$$


These $j$ equations are in fact $j$ linear equations in the unknowns $x_{r}=F^{(r-1)}(0)$, $r=1,2, \cdots, j$, and substituting for the $B_{i}$, they may be written

$$
\sum_{i=1}^{s+k} \gamma_{i} \sum_{r=1}^{j} x_{r} \sum_{s=r}^{j}\left(\begin{array}{l}
j \\
s
\end{array}\right) \beta_{i}^{n-r}\left(-\beta_{i} / \lambda\right)^{s}=\delta_{n 1}
$$

where $\delta_{n 1}$ is the Kronecker delta, and for $i=1,2, \cdots, j+k$,

$$
\gamma_{i}=\frac{\left(1+\beta_{i} / \mu\right) e^{\beta_{2} R}}{\left(1-\beta_{i} / \lambda\right)^{j-1}\left[\left(1-\beta_{i} / \lambda\right) k / \mu-\left(1+\beta_{i} / \mu\right) j / \lambda\right]} .
$$

The set of linear equations may be written in matrix form as $\boldsymbol{A B C} \boldsymbol{x}=\boldsymbol{\delta}_{1}$ where the $j$-rowed vectors $X$ and $\delta_{1}$ have elements $x_{r}$ and $\delta_{r 1}$, the typical $(r, s)$ th elements of $\boldsymbol{A}, \boldsymbol{B}, \boldsymbol{C}$ are

$$
a_{r s}=\gamma_{s} \beta_{s}^{r-1}, b_{r s}=\beta_{r}^{\sharp}, c_{r s}=\left(\begin{array}{l}
j \\
r
\end{array}\right)(-\lambda)^{-r} \text { if } r \geqq s, c_{r s}=0
$$

otherwise, and $\boldsymbol{A}$ is $j \times(j+k), \boldsymbol{B}$ is $(j+k) \times j, \boldsymbol{C}$ is $j \times j$ and lower triangular; $\boldsymbol{A} \boldsymbol{B}$ is $j \times j$ and symmetric. By inspection, $\boldsymbol{C}$ is of full rank, and intuitively we should expect $\boldsymbol{A B}$ to be of full rank (i.e., of rank $j$ ). Assuming the latter, a unique non-zero solution $\boldsymbol{x}$ exists, and the constants $B_{i}$ are finally computed from

$$
B_{i}=\gamma_{i} e^{-\beta, K} \sum_{r=1}^{j} \beta_{i}^{-\tau} x_{r} \sum_{s=r}^{j} \beta_{i}^{s} c_{s}
$$

observing that $\sum_{s=r}^{j} \beta_{i}^{s} c_{s}$ is the typical $(i, s)$ th element of the $(j+k) \times j$ matrix $\boldsymbol{B C}$.

We note that the probability of zero waiting-time is

$$
W(0)=\sum_{i=1}^{j+k} B_{i}
$$

The following moments for $w$ may be found, supposing $\beta_{1}=0$ to be the zero of $P(z)$ at the origin:

$$
\begin{aligned}
& E(w)= K\left(1-B_{1}\right)+\sum_{i=2}^{j+k}\left(1-e^{\beta_{i} K}\right) B_{\imath} / \beta_{i} \\
& \operatorname{Var}(w)=\sum_{i=2}^{++k}\left(e^{\beta_{i} K}-1\right) B_{\imath} / \beta_{\imath}^{2}-[E(w)]^{2}+2 K\left[E(w)-\sum_{\imath=2}^{j+k} B_{i} / \beta_{i}\right] \\
&+K^{2}\left(B_{1}-1\right) .
\end{aligned}
$$

It may be noted also that the constants $B_{\imath}$ here are dependent on the boundary values $F^{(n)}(0)$, whereas in the example $M / E_{k} / 1$ the constants $A_{i}$ are independent of $F(0)$.

Having obtained a solution for $E_{l} \mid E_{k} / 1$, there remains the question (and we leave it open) of the relation between this case and $D / E_{k} / 1$ (or by 
duality, $\left.E_{j} / D / 1\right)$. It may be conjectured that since $E_{,} \rightarrow D$ as $j \rightarrow \infty$ (such that $j / \lambda$ remains constant), so this behaviour should be reflected in the function $W(x)$. Results in this direction should then link up with Prabhu's work [14].

\section{The busy period and loss-free sequences}

We consider finally the question of the busy period of the server, and the related (though not dural) concept of the number of customers in succession who do not leave the system impatiently. In the dam model analogue, the corresponding ideas are of the number of years in succession in which the dam does not run dry, and in which it does not overflow, respectively.

Define $\left\{p_{n}(x, K)\right\}$ as the conditional distribution of the number of customers served in a busy period given that the first customer has waitingtime $x, 0 \leqq x \leqq K$. Then the distribution of the number of customers served in a busy period is $\left\{p_{n}(0, K)\right\}$, and for $n=1,2, \cdots$,

$$
p_{n}(x, K)=\operatorname{Pr}\left(w_{i}>0, i=1,2, \cdots, n-1, w_{n}=0 \mid w_{0}=x\right) .
$$

We use the generating function

$$
P(x, K, z)=\sum_{n=1}^{\infty} p_{n}(x, K) z^{n-1}
$$

noting that $P(x, K, 0)=p_{1}(x, K)$.

By relating $p_{n+1}(x, K)$ to $p_{n}(x, K)$ we derive an integral equation for $P(x, K, z)$ which in the case of a negative exponential service time distribution admits a formal solution via an integro-differential equation and extension of the range of definition of $P(x, K, z)$ to all $x \geqq 0$, both of which techniques we have used earlier.

The case $K \rightarrow \infty$ reduces to the moving server problem with an absorbing barrier (see § 2) for which it has been shown that in $M / G / 1$ (see [6] and [12]) $P(x, z)=\lim _{K \rightarrow \infty} P(x, K, z)$ satisfies

$$
P(x, z)=\exp \left\{-\lambda x\left[1-z \int_{0}^{\infty} P(t, z) d B(t)\right]\right\}
$$

with $A(x)=1-e^{-\lambda x}, x \geqq 0, \lambda>0$.

We state our result in

THEOREM 4. In the queueing system GI/G/1 with uniformly limited total queweing-time the conditional busy period distribution generating function $P(x, K, z)$ satisfies the equation 
where

$$
\begin{aligned}
P(x, K, z)-P(x, K, 0)= & z[1-B(K-x)] \int_{0}^{K} P(K-t, K, z) d A(t) \\
& +z \int_{x}^{K} d B(y-x) \int_{0}^{y} P(y-t, K, z) d A(t)
\end{aligned}
$$

$$
P(x, K, 0)=1-A(K)+\int_{x}^{K} B(t-x) d A(t) .
$$

Proof. Define $G(y, x)=\operatorname{Pr}\left(w_{1} \leqq y \mid w_{0}=x\right)$. Then from (6).

$$
\begin{aligned}
G(y, x) & =1-A(K-y)+\int_{y}^{K} B(u-x) d A(u-y) \\
& =1-[1-B(K-x)] A(K-y)-\int_{y}^{K} A(u-y) d B(u-x) .
\end{aligned}
$$

Since $P(x, K, 0)=p_{1}(x, K)=G(0, x)$, (25) follows immediately.

From the definition of $p_{n}(x, K)$, for $n=2,3, \cdots$,

$$
\begin{aligned}
p_{n}(x, K)= & \int_{0+}^{K} \operatorname{Pr}\left(w_{i}>0,\right. \\
& \left.\quad i=2,3, \cdots, n-1, w_{n}=0 \mid w_{1}=y\right) d_{v} G(y, x) \\
= & \int_{0+}^{K} p_{n-1}(y, K) d_{v} G(y, x)
\end{aligned}
$$

and on forming generating functions,

$$
\begin{aligned}
& P(x, K, z)-P(x, K, 0)=z \int_{0+}^{K} P(y, K, z) d_{y} G(y, x) \\
& \quad=z\left\{P(K, K, z) G(K, x)-P(0, K, z) G(0, x)-\int_{0+}^{K} G(y, x) d_{y} P(y, K, z)\right\} .
\end{aligned}
$$

Now $G(K, x)=1$, and on substituting for $G(y, x)$ and $G(0, x)$ and changing the order of integration once again, $(24)$ is deduced. This proves the theorem.

Example. $G I / M / 1$.

Substituting $B(x)=1-e^{-\mu x}, x \geqq 0, \mu>0$, in (24) and (25) leads formally to the solution (27) below. We denote the derivative of $P(x, K, z)$ with respect to $x$ by $P^{\prime}(x, K, z)$.

First, from (25),

$$
P(x, K, 0)=1-A(x)-\int_{x}^{K} e^{-\mu(t-x)} d A(t)
$$

whence $\mu P(x, K, 0) \rightarrow P^{\prime}(x, K, 0)=\mu[1-A(x)]$.

Next, from (24),

$$
\begin{aligned}
P(x, K, z)-P(x, K, 0)= & z e^{\mu x}\left[e^{-\mu K} \int_{0}^{K} P(K-t, K, z) d A(t)\right. \\
& \left.+\int_{x}^{K} \mu e^{-\mu y} d y \int_{0}^{v} P(y-t, K, z) d A(t)\right]
\end{aligned}
$$

and by differentiating and substituting for $\mu P(x, K, 0)-P^{\prime}(x, K, 0)$,

$$
P^{\prime}(x, K, z)=\mu\left[P(x, K, z)+A(x)-1-z \int_{0}^{x} P(x-t, K, z) d A(t)\right] .
$$


Assume now that there exists a function $P(x, K, z)$ satisfying this equation for all $x \geqq 0$ (and not merely $0 \leqq x \leqq K$ ), and that its transform $\hat{P}(\theta, K, z)=\int_{0}^{\infty} e^{-\theta x} P(x, K, z) d x$ exists for $\theta$ in some half-plane. Writing $\alpha(\theta)$ for the Laplace-Stieltjes transform of $A(x)$, we finally obtain

$$
P(\theta, K, z)=\frac{\theta P(0, K, z)-\mu[1-\alpha(\theta)]}{\theta\{\theta-\mu[1-z \alpha(\theta)]\}} .
$$

Inversion of $\hat{P}(\theta, K, z)$ yields $P(x, K, z)$ up to the function $P(0, K, z)$ which is found from (26) with $x=K$, namely,

$$
P(K, K, z)=1-A(K)+z \int_{0}^{K} P(K-t, K, z) d A(t) .
$$

For example, in the system $M / M / 1$ with $A(x)=1-e^{-\lambda x}$, we substitute $\alpha(\theta)=(1+\theta / \lambda)^{-1}$ in $(27)$ and seek the inverse of

$$
\frac{(\lambda+\theta) P(0, K, z)-\mu}{\theta^{2}-(\mu-\lambda) \theta-\lambda \mu(1-z)} \text {. }
$$

This yields $P(x, K, z)=C_{1} e^{c_{1} x}+C_{2} e^{c_{2} x}$ where $c_{1}, c_{2}$ are the zeros of the denominator (and they are distinct provided $\lambda \neq \mu$, i.e., $\rho=\lambda / \mu \neq 1$, else $\left.P(x, K, z)=\left(C_{1}+C_{2} x\right) e^{c_{1} x}\right)$, and $C_{1}, C_{2}$ are functions of $P(0, K, z)$. Using the boundary condition (28) leads to

$$
P(0, K, z)=\frac{(\mu-\lambda) \tanh \left(\frac{1}{2} a K\right)+a}{[\mu+\lambda(1-2 z)] \tanh \left(\frac{1}{2} a K\right)+a}
$$

where $a=\sqrt{ }\left[(\lambda+\mu)^{2}-4 \lambda \mu z\right]$ and finally,

$$
\begin{aligned}
P(x, K, z)= & a^{-1} e^{\frac{1}{2(\mu-\lambda) x}} \cosh \left(\frac{1}{2} a x\right)\left\{P(0, K, z)\left[(\mu+\lambda) \tanh \left(\frac{1}{2} a x\right)+a\right]\right. \\
& \left.-2 \mu \tanh \left(\frac{1}{2} a x\right)\right\}
\end{aligned}
$$

The mean $m(x, K)$ of the distribution $\left\{P_{n}(x, K)\right\}$ is evaluated by

$$
\begin{aligned}
m(x, K) & =1+\left.\frac{\partial}{\partial z} P(x, K, z)\right|_{z=1} \\
& =\frac{\mu(1+\lambda x)}{\mu-\lambda}-\frac{\lambda e^{-(\mu-\lambda) K}\left[\mu e^{(\mu-\lambda) x}-\lambda\right]}{(\mu-\lambda)^{2}}
\end{aligned}
$$

so the mean number of customers in a busy period, given by $m(0, K)$, is

$$
m(0, K)=\frac{1-\rho e^{-(1-\rho) \mu K}}{1-\rho}
$$

which in the case $\rho<1, K \rightarrow \infty$, reduces to $1 /(1-\rho)$, the well-known result for the standard system $M / M / 1$. 
The related distribution $\left\{q_{n}(x, K)\right\}$ of the number of customers in succession who do not leave the system impatiently, given that the first customer has waiting-time $x$, is defined for $n=0,1,2, \cdot \cdot$ and $0 \leqq x \leqq K$ by

$$
q_{n}(x, K)=\operatorname{Pr}\left(w_{\imath}+s_{\imath} \leqq K, \quad i=0,1, \cdots n-1, w_{n}+s_{n}>K \mid w_{0}=x\right) .
$$

Putting $Q(x, K, z)=\sum_{n=0}^{\infty} q_{n}(x, K) z^{n}$, we may proceed as in the proof of Theorem 4 and deduce that for $0 \leqq x \leqq K, Q(x, K, z)$ satisfies

$Q(x, K, z)-Q(x, K, 0)=z \int_{x}^{K} d B(y-x)\left[\int_{0}^{y} Q(y-t, K, z) d A(t)+Q(0, K, z) \int_{y}^{\infty} d A(t)\right]$.

The distribution $\left\{r_{n}(x, K)\right\}$ which we define by

$$
r_{n}(x, K)=\operatorname{Pr}\left(v_{\imath}+t_{i} \leqq K, \quad i=0,1, \cdots, n-1, v_{n}+t_{n}>K \mid v_{n}=x\right)
$$

is the dual (cf. $\S 3$ ) of $\left\{q_{n}(x, K)\right\}$, and its generating function $R(x, K, z)=$ $\sum_{n=0}^{\infty} \gamma_{n}(x, K) z^{n}$ is related to $P(x, K, z)$ by

$$
P(x, K, z)=\int_{x}^{K} R(K-y, K, z) d B(y),
$$

as may be shown by the methods of this section.

\section{Acknowledgements}

I am grateful to Mr. P. D. Finch for helpful discussion on the work reported here, and to Mr. J. F. C. Kingman for much useful criticism of an earlier version of the paper.

The work was carried out during the tenure of a Commonwealth Government Post-Graduate Award.

\section{References}

[1] Barrer, D. Y., Queueing with impatient customers and indifferent clerks, Operat. Res. 5 (1957), 644-649.

[2] Barrer, D. Y., Queueing with impatient customers and ordered service, Operat. Res. 5 (1957), 650-656.

[3] Doob, J. L., Stochastic Processes. Wiley, New York (1953).

[4] Finch, P. D., A limit theorem for Markov chains with continuous state space, J. Austral. Math. Soc. 3 (1963), 351-358.

[5] Ghosal, A., Emptiness in the finite dam, Ann. Math. Statist. 31 (1960), 803-808.

[6] Karlin, S., Miller, R. G. and Prabhu, N. U., Note on a moving single server problem, Ann. Math. Statist. 30 (1959), 243-246.

[7] Kendall, D. G., Some problems in the theory of queues, J. R. Statist. Soc. B 13 (1951), $151-185$.

[8] Kovalenko, I. N., A study of the queueing system $M / M / s$ with ordered service and limited time of staying in the system (In Russian), Ukrain. Mat. Z. 12 (1960), 471-476.

[9] Kovalenko, I. N., Some queueing problems with restrictions (In Russian), Teor. Veroyat. Primen. 6 (1961), 222-228. 
[10] Lindley, D. V., The theory of queues with a single server, Proc. Camb. Phil. Soc. 48 (1952), 277-289.

[11] Loynes, R. M., The stability of a queue with non-independent inter-arrival and service times, Proc. Camb. Phil. Soc. 58 (1962), 497-520.

[12] McMjllan, B. and Riordan, J., A Moving single server problem, Ann. Math. Statist. 28 (1957), 471-478.

[13] Moran, P. A. P., A probability theory of dams and storage systems, Aust. J. Appl. Sci. 5 (1954), 116-124.

[14] Prabhu, N. U., On the integral equation for the finite dam, Quart. J. Math. (2). 9 (1958), $183-188$.

[15] Prabhu, N. U., Application of storage theory to queues with Poisson arrivals, Ann. Math. Statist. 31 (1960), 475-482.

[16] Saaty, T. L., Elements of Queueing Theory with Applications. McGraw-Hill, New York (1961).

[17] Takacs, L., Investigation of waiting-time problems by reduction to Markov processes, Acta Math. Acad. Sci. Hung. 6 (1955), 101-129.

[18] Takacs, L., Introduction to the Theory of Queues. Oxford University Press, New York (1962).

Department of Statistics, University of Melbourne, and

Statistical Laboratory, University of Cambridge. 